\title{
Technological Advances in Drug Susceptibility Testing-Diagnosis of Tuberculosis
}

\author{
E. Vanithadevi* \\ Department of Microbiology, Karpaga Vinayaga Institute of Medical Science, T.N., India \\ *Corresponding author
}

\section{A B S T R A C T}

\begin{tabular}{|l|}
\hline K e y w o r d s \\
Technological Advances, \\
$\begin{array}{l}\text { Drug Susceptibility, } \\
\text { Testing, Diagnosis of } \\
\text { Tuberculosis }\end{array}$ \\
\hline Article Info \\
\hline $\begin{array}{l}\text { Accepted: } \\
\text { 24 May 2018 } \\
\text { Available Online: } \\
\text { 10 June 2018 }\end{array}$ \\
\hline
\end{tabular}

Nowadays the technology has rapidly advanced in the detection of mycobacterium tuberculosis and drug susceptibility clinical sample. The examination that include the advances microscopic, in viro-culture and application in molecular base. In developing countries the world health organization is played a role for evaluating and developing the technology of their efficiency, feasibility at present in India, natural network and designed microbiology centre, and also intermediate reference laboratories implemented by the revised national tuberculosis control programme RNTCP. Advance staining of sputum smears in microscopy technology that includes fluorescent microscopy and vital fluorescent. The turnaround time, if there is smear negative samples, and permits detection of resists mutants simultaneously. The detection of drug resistance in molecular base has reduced further TAT, and also the cartridge based nucleic acid tests with its convenience results, poised appears to become tuberculosis (TB) diagnosis provided, testing cost is reduce especially use of private diagnostic. This paper is reviews of recent technology adopted in diagnosis of TB.

\section{Introduction}

The antimicrobial susceptibility testing performance of antimicrobial susceptibility test. In 2016, 9.43 million cases of tuberculosis (TB) was report worldwide, in that the indented were estimate 2.6 million samples in India. The testing objective is to detect possible drug resistance in general pathogens and to ensure suscepbility to drugs to the particular infections. The general testing methods are directly detected the activity growing bacilli and indirectly detect the immune response bacilli. The manual approach that provide flexibility and possible to cost savings. In both method have strength and weaken, including organisam that may be processing tested by method. The (WHO) world health organization global (TB) reported in 2017. TB killed 1 million people ( 0.8 to 12 ) in that HIV negative 0.32 million HIV positive in 2016. The main 30 TB burden countries accometed in $92 \%$ of all estimated cases in the world. The MR-TB, mycobacterian tuberculosis is having complete isolates with a viu-resistance. The anti- $\mathrm{Tb}$ drugs first time resistance with vitro resistance to fluoroquinolone kanamycin (KM) [Pai and Sharma, 2007], a mikacin (AMK) as extensively drug resistance TB 
(XDR). The emerging of anti-microbial resistance and the rotation able for suscepbility testing. In clinical laboratory antimicrobial susceptibility test is important to chosen empirical agents. The challenging of MDR emergence, diagram

Direct identification of disease is included microbiology test, culture media antigen of identification and identification no factor.

\section{Direct Detection Method}

\section{Microscopy-sputum smear}

Sputum is often the first TB test to be used in countries with high rate of TB infections. It is a basics for diagnosis of TB. The general use of acid fast staining using carbol fuschin and fluorochrome dyc cuuputum mearamine [Scientists find cure for extreme TB strain, 2009]. Direct sputum smear microbiology. Direct sputum smear microbiology is the most widely used means for diagnosis pulmonary TB and is available in most primary health centre laboratories at health centre lave. Smear microbiology is been costly and inconvenient and patients, who have to make multiple visits to health lab to submit multiple sputum smear specimens or several days. It has been shown conclusively good quality microscopy of two consectutive sputum specimen identificaties the majority $95-98 \%$ of smear positive TB patent.

Conventional case finding approaches initial improve microscopy examination of spot morning, sputum specimens (or) examination of spot-morning spot each sputum result will give 3-4 days later, so patient wait at health centre. During the test at least $2 \mathrm{ml}$ specimen should be examined. The result were documented first day specimen detection percentage and the second day detection percentage. The diagnosis centre are do not follow routine type microcopy because they differentiate between live and dead bacilli. RNTCP has revised the the diagnostic procedure to use for nuclic acid specimen magnification detection base on the cartage based (CBWAAT) technology.

\section{Front loaded microscopy}

Diagnosis of pulmonary TB by conventional smear microscopy requires patient attendance on 2 consecutive days. We investigated whether collecting sputum on the spot for smear microbiology on the days the patient presented was sensitive and specific for diagnosis as spot morning spot morning presumptive pulmonary TB cases submitted three sputum specimen on the spot on early morning specimen on the spot an early morning sputum. An additional specimen was collected $1 \mathrm{~h}$ after the first spot on the $1^{\text {st }}$ day of consultation. Sputum smear more made on clear, Greece- free slides and stained using the ziehl-nelson technique. All stained smears were read at 1000X magnification and graded accordingly with the one or more acid fast bacilli high power field was considered positive. The yields of the standard scheme. Two of the paired observational studies carried out.

\section{Sodium hypochlorite microscopy}

In revised national tuberculosis control program microscopic examination of sputum for AFB plays an important role in the initial diagnosis of tuberculosis bacillary concentration after decontamination and liquefaction by $5 \%$ of sodium hypochlorite [ICMR, 2002]. Sensitivity and safely for handling of specimen. In this cross-sectional, prospective study, carried out, patient were include accordingly to RNTCP guideline while another set was prepared by concentration after decomposition with 5\% sodium hypochlorite. There is a statically significant rise in smear positive case after 
considering its low cost decontaminating and liquesfaction properties with better sensitivity, this method is safe and can be vital importance at least for smear negative cases.

\section{Vital fluorescent staining}

The performance of FDA vital staining method in detecting viability of and fast bacilli in comparison with culture and also its correlation with conventional day suscepbility testing results for isoni acid [The tuberculosis $\mathrm{x}$ factor, 2006; Katoch, 2004]. The study was conducted sputum samples of patients taking TB treatment were tested by vital staining. FDA microscopy can be used as a simple and repaired TB treatment monitoring tool in resource limited setting to identify those patient requiring immediate culture. The FDA is a vital stain with the technique based on the principle of intracellular FDA hydrolysis. The study was under taken determine the performance of FDA vital staining in viability of acid fast bacilli (AFB) in rapid tool for identifying species of culture isolates.

\section{Fluorescent microscopy with light- emitting diodes mode}

The general conventionally used fluorescence microscopy (FM) the quartz -halogen lamp with high percentage mercury vapour lamp is expensive they needs experts to operate [Das et al., 2003; Jadaun et al., 2007]. The results showed that the accuracy of LED microscopy reference std. light emitting diode fluorescence microscopy has been shown to be more sensitive than conventional bright field microscopy using ziehl-nelson stain in detecting sputum smear positive tuberculosis in controlled laboratory conditions. The proportion of presumptive TB patient (PTP) found sputum smear positive increased by $30 \%$ from $13.6 \%$ use of LED-Fm significantly increased the proposition of smear positive cases among presumptive.

\section{Recent advances in microscopic}

In fast moving world the technology using a new imaging technique described in Science on living cells can be filmed in high-resolution and 3-D, producing stunning videos of their fully animated worlds. "Studying the cell on a coverslip is like watching a lion in the zoo you're not exactly seeing their native behaviors," says physicist Eric Betzig. Using the new microscope "is like watching the lion chase an antelope on, you're finally seeing the true nature of cells."

Various techniques such as sandwich- and inhibition enzyme-linked immunosorbent assay (ELISA), latex agglutination and reverse passive haemagglutination have been utilized for the detection of different mycobacterial antigens in cerebrospinal fluid and in pleural fluid. These assays were found to be successful in detecting the whole antigens (viz. mycobacterial sonicates and extracted glycolipids, PPD) as well as various antigenic fractions (Ag5 or $38 \mathrm{kDa}, \mathrm{AgA60}$, 45/47kDa Ag, Ag Kp90, 30kDaAg, P32 Ag, cord factor or trehalose dimycolate and lipoarabinomannan LAM) detecting the antigens as low as 3-20 ng/ ml. of the samples [ICMR, 2002]. Also antigen capture ELISA employing monoclonal antibody and a dipstick test have been found quite sensitive and specific for detection of LAM in sputum and to some extent, in urine samples too. Immuno-chromatographic techniques (ICT) are now commercially available for rapid diagnosis of TB by the antigen detection.

\section{Culture}

Sputum culture is a test to detect and identify bacteria or fungi infect the lungs. The culture test involves studying bacteria by growing the bacteria on different substance. This is to found the particular bacteria a present in that. 
The bacteria are usually contained in a sputum sample plates from the patient suspected of having TB [Jadaun et al., 2007; Krishnamurthy et al., 2002].

Mycobacterium tuberculosis are called acid fast bacilli, because after an acid wash, the susceptibility test means testing of resistant $t$ any TB drugs which is MDR TB or XDR TB.TB drug resistance testing involves culturing TB bacteria in presence of anti $\mathrm{TB}$ drugs. If the bacteria grow it means that the bacteria are resistant to the drugs.

Culturing and identifying M.tuberculosis bacteria proving a definite very accurate diagnosis of TB. This is in contrast to other TB test where the lack of accuracy problem. Culturing bacteria to test for $\mathrm{TB}$ is much more complex and expensive to carry out than sputum smear microbiology. This is because it requires specific equipment and laboratory facilities.

Testing and diagnosing TB using culture can also take weeks because of the slow growth of the bacilli. TB cultures are performed only at national reference laboratories or in hospital laboratories. Depend upon bacteria grow in the culture, the oxygen by florescence. Oxygen is utilized causing when placed under UV light.

In the manual system, a technician visually identifies fluoresces. Automated system can benefit laboratories with a high work load, and provide. Standard liquid media are more process to contamination than solid media, leading to invalid results unless carefully controlled. An automated machine must be maintained, required technical support from the manufacturer and agent. The diagnosis can be taken for a 9 days for a smear positive specimen to 16 days for smear- negative specimen. The negative result will be given by 40days after initial culture.

\section{Fast identification instrument for species of} culture isolates

Another important step towards early diagnosis is rapid identification of mycobacterial isolates from conventional cultures or rapid methods like BACTEC and others [Krishnamurthy et al., 2002; Katoch, 2008]. Traditionally biochemical tests are used for identification of mycobacteria. These are, however, time consuming and sometimes ambiguous results are obtained. To overcome these limitations chemical methods based on lipid profiles hybridization with specific gene probes, Polymerase chain reaction- restriction fragment length polymorphism (PCR-RFLP) methods such as gene for hsp $65 \mathrm{kDa}$ protein, $\mathrm{katG}$, and rRNA genesand sequencing of $16 \mathrm{~S}$ rRNA have been described.

Analysis of lipid profiles: Mycobacteria have characteristic lipid profiles. These lipid profiles can be analysed by HPLC or HPTLC13 and quick identification of mycobacterial isolates can be done. This strategy has been successfully used in several laboratories.

DNA probes: Based on information about specific gene sequences well defined oligonucleotide probes for identification of various clinically relevant mycobacteria have been developed and are readily available.

These include probes for identification of $\mathrm{M}$. tuberculosis, M. avium and several other mycobacteria. These probes are being used in several countries for rapid confirmation of the identity of mycobacterial isolates. When used along with newer methods of detection of the early growth (such as BACTEC, Septi-Chek, MGIT) these are of great help in rapidly confirming the diagnosis as identity of isolate can be established within 1 to 2 days with gene probes as compared to much longer time required with classical biochemical tests. 
Ribosomal rRNA based probes: In recent years, ribosomal RNA gene region has been extensively explored for designing systems for ribosomal DNA fingerprinting and for development of probes/as well as gene amplification assays for various of Katoch: Rapid Diagnostic Methods for Tb 420 Indian J Med Res, October 2004 mycobacterial species including M. tuberculosis, M. leprae and $\mathrm{M}$. avium etc. These probes target rRNA, ribosomal DNA, spacer and flanking sequences. Commercially available rRNA based probes have been reported to be useful for quick identification of mycobacterial isolates. These probes were earlier radiolabeled but have now been developed into chemiluminescent techniques.

Gene amplification methods for identification: Techniques may also be used for confirmation of the identity of isolates but the problem of carry-over from the original inoculum needs to be kept in mind. Different strategies to identify the isolates from cultures and also directly from the clinical specimens have been described. These include amplification of specific gene regions followed by hybridization with species specific probes6,7, sequencing and RFLP analysis such as hsp $65 \mathrm{kDa}$ gene, katG and rRNA genes, a CJIL system under publication) have been described. These PCRRFLP assays help in quick identification of pathogenic mycobacteria including $M$. tuberculosis from the culture isolates as well as directly from the clinical specimens. While PCR-sequencing approach can be applied by reference laboratories the hybridization and RFLP approaches are easily practicable in clinical mycobacteriology laboratories.

Gene amplification methods for direct detection of M.tuberculosis sequences from clinical specimens: Gene amplification techniques have made a major impact on the diagnosis of mycobacterial diseases. These methods may be classified as those based on polymerase chain reaction (PCR) and others based on isothermal amplification reactions. Gene amplification techniques are highly sensitive and under optimum conditions may detect 1-10 organisms. If systems are adequately standardized, evaluated and precautions for avoiding the contamination are taken, these assays can play a very useful role in early confirmation of diagnosis in paucibacillary extra-pulmonary forms of tuberculosis.

\section{Antigen detection}

Tests that detect TB antigens in clinical specimens, if accurate, could provide direct evidence of active TB [Sekar et al., 2008; Hoek et al., 2008; Hain Lifesceince $\mathrm{GmbH}$, 2007], thus allowing for the immediate start of TB treatment. In comparison with conventional diagnostics, antigen detection tests appear to offer several advantages: immunochromatographic tests (dipsticks are an example), are rapid (results may be available within minutes) and easy to operate. If developed into a point-ofcare test, an antigen detection test could extend TB diagnosis to remote community health facilities. An antigen detection test using a specimen such as urine would be particularly attractive in children, who may have difficulty providing sputum. Finally, in patients suspected of extrapulmonary $\mathrm{TB}$, an antigen detection test might prevent the use of more invasive tests.

\section{Molecular detection: Nucleic acid amplification tests}

Molecular diagnostics is a collection of techniques used to analyse biological markers in the genome and proteome the individual's genetic code and how their cells express their genes as proteins by applying molecular biology to medical testing. The technique is 
used to diagnose and monitor disease, detect risk, and decide which therapies will work best for individual patients.

A nucleic acid test NAT is a technique utilized to detect a particular nucleic acid, virus, or bacteria which acts as a pathogen in blood, tissue, urine, etc. NAT system is a little different from other tests in that it detects genetic materials rather than antigens or antibodies. Detection of genetic materials allows an early diagnosis of a disease because the detection of antigens requires time for antigens to appear in the bloodstream. [Pai and Sharma, 2007] Since the amount of a certain genetic material is usually very small, NAT includes an amplification step of the genetic material.

These techniques also have an important role in diagnosis of tuberculosis, Currently three methods exist; the first two being WHO recommended [Hain Lifesceince $\mathrm{GmbH}$, 2007]:

Cartridgebased NAAT (CBNAAT)

Line probe assay (LPA)

Loop mediated amplification (LAMP).

\section{Cartridgebased NAAT (CBNAAT)}

Cartridge based nucleic acid amplification test (CB-NAAT, GeneXpert,) is an automated cartridge-based molecular technique which not only detects Mycobacterium Tuberculosis but also rifampicin resistance within two hours and has been endorsed by WHO as an initial diagnostic test in children suspected of having tuberculosis both in pulmonary and specific forms of extra pulmonary tuberculosis. Globally the use of rapid molecular tests is increasing. Despite advances in diagnostics, a considerable proportion of the TB cases reported to $\mathrm{WHO}$ is still clinically diagnosed rather than bacteriological confirmation. So hereby we are reporting case series where CB-NAAT helped us in early diagnosis and effective management.

Our hospital is a tertiary care hospital and a designated medical centre for Directly observed treatment, short-course (DOTS) but CB- NAAT testing facility is not available in our centre. We transported the samples to the nearest MDR treatment centre in Mangalore where CB-NAAT testing is done free of cost by the government

The sensitivity was $99.8 \%$ for smear and culture- positive cases and $90.2 \%$ for smearnegative, culture positive cases. The sensitivity of a single direct MTB/RIF test for culture confirmed TB was $92.2 \%$ and rose to $96.0 \%$ with the additional testing of a pelleted sample. For the detection of smear negative, culture positive TB, the sensitivity of the assay was $72.5 \%$ for one test, $85.1 \%$ for two tests and $90.2 \%$ for three tests. The estimated specificity was $99.2 \%$ for a single direct MTB/RIF test, $98.6 \%$ for two MTB/RIF tests and $98.1 \%$ for three MTB/RIF tests. The MTB/RIF test correctly detected RIF resistance with a sensitivity of $99.1 \%$ and $100 \%$ specificity.

\section{Line probe assay (LPA)}

Mycobacterium tuberculosis complex may sometimes not be detected in sputum samples of suspected multidrug-resistant tuberculosis (MDR-TB) patients by line probe assay (LPA) even though they are smear positive for acid-fast bacilli (AFB). This retrospective analysis was attempted to understand and document our experience with LPA for detection of $\mathrm{M}$. tuberculosis complex and diagnosis of MDR-TB under programmatic conditions. The method for using for tuberculosis One thousand two hundred and ninety four sputum samples of MDR-TB suspects that were smear positive for AFB, 
and received from February to November 2013, were tested by LPA for the presence of M. tuberculosis complex and resistance to isoniazid (INH) and rifampicin as per the diagnostic mandate of an accredited reference laboratory. As per the mandate, those samples that were negative for $M$. tuberculosis complex were cultured, and the growth again tested by LPA. A retrospective analysis of the results was carried out. Results were obtained in this method of testing is LPA is a robust technique for diagnosis of drug-resistant TB that has provided the basis for rapid and effective control of drug-resistant TB in India. While the reasons for concomitantly negative LPA and culture results of smear-positive sputum samples from MDR-TB suspects may be many, the possible presence of nontubercular mycobacteria in these samples and the likelihood of inappropriate therapy in these patients cannot be ruled out. Addition of culture to the diagnostic algorithm may enhance the diagnostic yield.

\section{Loop mediated amplification (LAMP)}

LAMP is an isothermal nucleic acid amplification technique. In contrast to the polymerase chain reaction (PCR) technology in which the reaction is carried out with a series of alternating temperature steps or cycles, isothermal amplification is carried out at a constant temperature, and does not require a thermal cycler.

In LAMP, the target sequence is amplified at a constant temperature of $60-65^{\circ} \mathrm{C}$ using either two or three sets of primers and a polymerase with high strand displacement activity in addition to a replication activity. Typically, 4 different primers are used to identify 6 distinct regions on the target gene, which adds highly to the specificity. An additional pair of "loop primers" can further accelerate the reaction. [ICMR, 2002] Due to the specific nature of the action of these primers, the amount of DNA produced in LAMP is considerably higher than PCR based amplification.

Detection of amplification product can be determined via photometry for turbidity caused by an increasing quantity of magnesium pyrophosphate precipitate in solution as a byproduct of amplification. This allows easy visualization by the naked eye, especially for larger reaction volumes, or via simple detection approaches for smaller volumes. The reaction can be followed in real-time either by measuring the turbidity [Katoch, 2004] or by fluorescence using intercalating dyes such as SYTO 9. Dyes such as SYBR green, can be used to create a visible color change that can be seen with the naked eye without the need for expensive equipment, or a response that can more accurately be measured by instrumentation. Dye molecules intercalate or directly label the DNA, and in turn can be correlated to the number of copies initially present. Hence, LAMP can also be quantitative.

In-tube detection of DNA amplification is possible using manganese loaded calcein which starts fluorescing upon complexation of manganese by pyrophosphate during in vitro DNA synthesis. [Jadaun et al., 2007] Moreover, visual detection of the LAMP amplicons by the unaided eye was based on their ability to hybridize with the complementary goldbound ss-DNA and thus prevent the normal red to purple-blue color change that would otherwise occur by salt-induced aggregation of the gold particles. So, a LAMP method combined with amplicon detection by AuNP has advantages over previously other methods in terms of reduced assay time, amplicon confirmation by hybridization and use of simpler equipment (i.e., no need for a thermocycler, electrophoresis equipment or a UV trans-illuminator. 


\section{Indirect method of detection}

\section{Serological tests}

Most of the serological tests used in the past have low sensitivity particularly, in cases of smear- negative HIV- positive patients. Also these assays were expensive and very often have difficulty in differentiating between $\mathrm{M}$. tuberculosis (Mtb) and other mycobacterium species infections in the past.

Some of the tests that have been developed recently that overcame these limitations areELISA and the ICT employing antigens such as LAM, $38 \mathrm{kDa}$ or AgA60 in detection of specific antibodies. Also ELISA has been evaluated in detection of antibodies to superoxide dimutase, an important secretory protein of $\mathrm{M} \mathrm{tb}$. ICT are now commercially available for rapid diagnosis of TB by the antibody detection.

\section{Miscellaneous diagnostic methods}

Newer tests with defined mycobacterial antigens such as MPB64 patch test has been devised which in contrast to tuberculin testing (Mantoux) [Kulkarni and Madrasi, 2008], showed the ability to distinguish the sensitized individuals from those suffering from active disease. Further, ESAT-6 (6 kDa) is a specific antigen that induces IFN gamma production by $\mathrm{T}$ lymphocytes of the $\mathrm{TB}$ patients. The antigen is recognized by $\mathrm{T}$ lymphocytes of TB patients but not by the $\mathrm{T}$ lymphocytes of the BCG vaccinated, thus making the test quite specific and reliable Also estimation of nitric oxide and protein carbonyl in serum of TB patients have been reported recently and the levels correlated well with each other. These changes are, however a reflection of increased host's defense mechanism at the cellular level against the organism that requires to be examined further in relation to the specificity.
The diagnostic procedures essentially require certain features such as sensitivity, specificity and speed along with cost- effectiveness for its reliability and wider applications. Smear examination/ microscopy has its inherent limitations, therefore culture methods are essentially required to be utilized along with the former for better results. As such, culture methods still remain the gold standard for the diagnosis as well as in validating other diagnostic tests for TB. In contrast, molecular methods are highly useful particularly, in the extra- pulmonary cases, if special facilities are available. With advent of various ICT based tests specifically detecting different mycobacterium antigenic fractions as well as antibodies against some of the antigenic fractions which has greatly revolutionary the facilities now available in the diagnosis of TB. Also substantial progress has been made in bringing out simpler techniques that may be utilized in early diagnosis as well as detection of drug susceptibility of the isolated organism in near future. In absence of the facilities for carrying out the molecular assays, inclusion of the ICT based tests in detection of both the antigen and antibody along with smear examination and culture facilities may serve the purpose well in a particular setup.

\section{References}

Das R, Shrivastava K, Gupta $\mathrm{P}$ et al., Comparison of $\mathrm{E}$ test with MIC method of Lowenstein-Jensen medium for susceptibility testing of Mycobacterium tuberculosis. Curr Sci 2003; 85: 191193.

Editorial. The tuberculosis $\mathrm{x}$ factor. Lancet 2006; 6: 679.

Hain Lifesceince GmbH: Molecular Diagnostics. Diagnostics to facilitate control of tuberculosis. Clin Lab Invest 2007; 31: 27. 
Hoek KGP, Gay Van Pittican MC et al., Fluorometric assay for testing rifamipicin susceptibility of Mycobacterium tuberculosis complex. J Clin Microbiol 2008; 46: 1369- 1373.

ICMR. What is new in the diagnosis of tuberculosis? Part 1. Techniques for diagnosis of tuberculosis. ICMR Bull 2002; 32: 69-76

Jadaun GPS, Aggarwal A, Sharma $\mathrm{H}$ et al., Determination of ethambutol MICs for Mycobacterium tuberculosis and $\mathrm{M}$. avium isolates by Resazurin microtitre assays. J Antimicrob Chemother 2007; 60: 152-155.

Katoch VM. New generation methods for drug susceptibility testing for tuberculosis. Indian J Tuberc 2008; 55: 61- 63.

Katoch VM. Newer techniques for tuberculosis. Indian J Med Res 2004; 120: 418- 428.

Krishnamurthy A, Rodrigues C and Mehta AP. Rapid detection of rifamipicin resistance in Mycobacterium tuberculosis by plaque assay. Indian $\mathbf{J}$ Med Microbiol 2002; 20: 211- 214.

Kulkarni A and Madrasi NA. Relationship of nitric oxide and protein carbonyl in tuberculosis. Indian J Tuberc 2008; 55: 138- 144.

News item. Scientists find cure for extreme TB strain. Combination of two FDAapproved drugs blocks growth of TB strain. The Times of India, Pune Thursday April 9, 2009; p. 7.

Pai $\mathrm{M}$ and Sharma SK. Editorial- Are we providing quality care to our patients with tuberculosis? Indian J Med Res 2007; 125: 491- 497.

Sekar B, Selvaray AA, Ravi S et al., The utility of IS 6110 sequence based polymerase chain reaction in comparison to conventional methods in the diagnosis of extra-pulmonary tuberculosis. Indian $\mathrm{J}$ Med Microbiol 2008; 26: 352- 355 .

\section{How to cite this article:}

Vanithadevi E. 2018. Technological Advances in Drug Susceptibility Testing-Diagnosis of Tuberculosis. Int.J.Curr.Microbiol.App.Sci. 7(06): 3127-3135. doi: https://doi.org/10.20546/ijcmas.2018.706.367 